\title{
Can blazar flares be triggered by the VHE gamma-rays from the surrounding of a supermassive black hole?
}

\section{Piotr Banasinski*}

Department of Astrophysics, University of Lodz, Lodz, Poland

E-mail: p.banasinski@uni.lodz.pl

\section{Wlodek Bednarek}

Department of Astrophysics, University of Lodz, Lodz, Poland

E-mail: bednar@uni.lodz.pl

\begin{abstract}
It is suggested that the extremely fast $\mathrm{TeV}$ gamma-ray flare from the radio galaxy IC 310 might originate in the magnetosphere of a supermassive black hole. If such flares are produced also in the surrounding of SMHBs in blazars then VHE gamma-rays have to propagate in the radiation field of the jet before reaching an observer. We investigate the scenario in which VHE gamma rays initiate electromagnetic cascades in the synchrotron radiation field produced in the whole volume of the jet.
\end{abstract}

35th International Cosmic Ray Conference - ICRC2017-

10-20 July, 2017

Bexco, Busan, Korea

${ }^{*}$ Speaker. 


\section{Introduction}

Blazars are the sources known for their fast variability. Several of the blazar $\gamma$-ray flares are exceptionally short, close to or even smaller than the light-crossing time of their supermassive black hole (SMBH). The most extreme examples, with the time variability order of minutes, are PKS 2155-304 [1], Mrk 501 [2], IC 310 [3]. Such an extremely short time variability may indicate that $\gamma$-rays are produced in the vicinity of SMBH. This explanation is especially favorable for BL Lacs where the radiation from a broad line region and an accretion disk is less intense than in FSRQs. Therefore, the high energy (HE) $\gamma$-rays produced close to the SMBH may avoid absorption [4].

Several models are proposed for the production of very high energy (VHE) $\gamma$-ray photons in the vicinity of SMBH. First group of models, inspired by pulsar models, propose the existence of a vacuum gap acceleration e.g. [5], [6]. The second one assumes that in the magnetic field of the accretion disk or X-ray corona occurs a magnetic reconnection where electrons are accelerated to VHE energy, e.g. [7]. In third group, the magnetic reconnection occurs in the jet and creates a minijets [8]. Such models predict very hard spectra of emitted VHE $\gamma$-rays, which can be interpreted as flare events of AGNs.

However, in case of blazars where the angle between the jet axis and the line of sight is smaller than the opening angle of the jet, HE $\gamma$-rays have to propagate in the radiation field of the jet. They may be absorbed in collisions with low energy photons. The effect of the low energy radiation from the jet on the absorption of $\gamma$-rays is usually ignored, in contrast to the cases of the absorption on the radiation from an infrared torus, a broad line region and an accretion disk.

In the present paper, we investigate the effect of the absorption of HE $\gamma$-rays in radiation of the extended jet for the observed spectra assuming that VHE $\gamma$-rays are produced close to the jet base. Absorbed $\gamma$-rays initiate an electromagnetic cascades inside the jet volume creating electronpositron pairs. Such $\mathrm{e}^{ \pm}$pairs produce secondary photons which can significantly effect the initial spectra.

\section{Electromagnetic cascades within a jet}

The blazars are observed in different emission states. We propose scenario where a persistent and low-level radiation is produced in an extended parsec-scale jet. On the other hand, fast flares are caused by VHE $\gamma$-rays produced close to the jet base. These $\gamma$-rays propagate in a radiation field of the jet. They are absorbed on the low energy photons within the jet initiating Inverse Compton (IC) $\mathrm{e}^{ \pm}$pair cascades.

We focus on the sources which observation angle is smaller than the half-opening angle of the jet. Although the IC $\mathrm{e}^{ \pm}$pair cascades can occur in the whole volume of the jet, the time of active state is comparable to the time of the injection of the VHE $\gamma$-rays. If the absorption occurs efficiently in the part of the jet with length $\Delta z$, the short impulse of the high energy radiation will be observed during the time interval $\Delta t_{o b s} \simeq \Delta z / c\left(1-\cos \theta_{o b s}\right)$, where $\theta_{o b s}$ is the angle between the line of sight and the jet axis. 


\subsection{Inhomogeneous stationary jet}

We approximate the geometry of the jet by a truncated cone. The base of the jet is located at distance $z_{0}$. The radius at the jet base is fixed on $R_{0}=10 R_{S c h}$, where $R_{S c h}$ is the Schwarzschild radius. The radius of the jet changes with the distance according to $R_{o b s}(z) \simeq R_{0}+\phi z$, where $\phi$ is the half of the opening angle of the jet. Based on the radio observations of parsec-scale jets [9, 10], we relate Lorentz factor of the jet, $\Gamma_{j}$, to the jet opening angle with $\phi \approx 0.3 / \Gamma_{j}$.

It is more convenient to consider the processes inside the jet in its plasma rest frame. In such a frame, the geometry of the jet is described by $R_{j}\left(t^{\prime}\right)=R_{0}+\phi \Gamma_{j} \beta_{j} c t^{\prime}$, where $\beta_{j} c$ is the velocity of the plasma in the jet. The magnetic field in the jet is obtained by normalizing to its base. It is inversely proportional to the radius of the jet, $B\left(t^{\prime}\right)=B_{0}\left(R_{0} / R_{j}\left(t^{\prime}\right)\right)$.

High energy electrons are injected continuously in the large part of the jet. We do not indicate the details of the acceleration process. It could be caused by an acceleration on the shock waves like in internal shock models or by an acceleration on the magnetic field irregularities. However, we assume that the injection of relativistic electrons occurs at distance from $z_{a c c}$ to $z_{\text {end }}$. The spectrum of electrons is described by a power law with an exponential cutoff at energy $E_{\max }=\gamma_{\max } m c^{2}$, where $\gamma_{\max }$ is the characteristic maximum Lorentz factor of the electrons. This maximum Lorentz factor of the injected electrons is obtained from the comparison of the acceleration time scale, $t_{a c c}^{\prime}$, with the total cooling time scale, $t_{\text {cool }}^{\prime}$, where $t_{\text {cool }}^{\prime}$ includes the synchrotron, inverse Compton and adiabatic cooling processes. The power injected to the jet changes with distance along the jet according to $\propto 1 / R j$. Finally, $t_{a c c}^{\prime}$ is characterized by an acceleration coefficient $\eta$, i.e. $t_{a c c}^{\prime}=R_{L} / \eta c$, where $R_{L}$ is the Larmor radius of electrons.

The time evolution of the electron's distribution within the jet is described with the kinetic equation,

$$
\frac{\partial N_{e}^{\prime}\left(\gamma, t^{\prime}\right)}{\partial t^{\prime}}=\frac{\partial}{\partial \gamma}\left(\frac{\mathrm{d} \gamma_{t o t}}{\mathrm{~d} t^{\prime}} N_{e}^{\prime}\left(\gamma, t^{\prime}\right)\right)+Q_{i n j}\left(\gamma, t^{\prime}\right)
$$

where $N_{e}$ is the differential spectrum of electrons, $\mathrm{d} \gamma_{t o t} / \mathrm{d} t^{\prime}=\gamma / t_{\text {cool }}^{\prime}$ is the total cooling rate and $Q_{i n j}$ describes the injection rate of electrons. The kinetic equation is solved numerically with the method presented in [11]. It should be noted that the term $\mathrm{d} \gamma_{\text {tot }} / \mathrm{d} t^{\prime}=\gamma / t_{\text {cool }}^{\prime}$ needs information about a local photon field (because the inverse Compton cooling is included). For this reason, we adopt the generation method to calculate the steady distribution of electrons. In the first approximation, we consider only the synchrotron and the adiabatic cooling processes. In the next step, when the photon field is already known, we solve the kinetic equation including also the IC process. We repeat this procedure a few times, until a stable solution of electron spectrum is obtained.

The local density of low energy photons, $n_{p h}^{\prime}$, at the time $t^{\prime}$ (where $t^{\prime}$ corresponds to the distance between the jet base and the location of the slab $z^{\prime}$ ) depends on the conditions in the whole jet. For simplicity, we assume that photons at specific distance, $z^{\prime}$, have two possible directions. For photons produced at the bottom of the jet, the direction of photons is consistent with the jet axis (we call them a backward photons and represent by subscript + ); for photons produced above $z^{\prime}$, the direction of photons is opposite to direction of the jet axis (forward photons, represented by -). Above approximation is reasonable due to the longitudinal shape of the jet, i.e. $\left(z_{\text {end }}-z_{a c c}\right) \gg R_{j}$. Such a condition is fulfilled in the presented model. We find the local density of photons, $n_{ \pm}\left(\varepsilon^{\prime}, t^{\prime}\right)$, by solving the radiation transfer equation, 

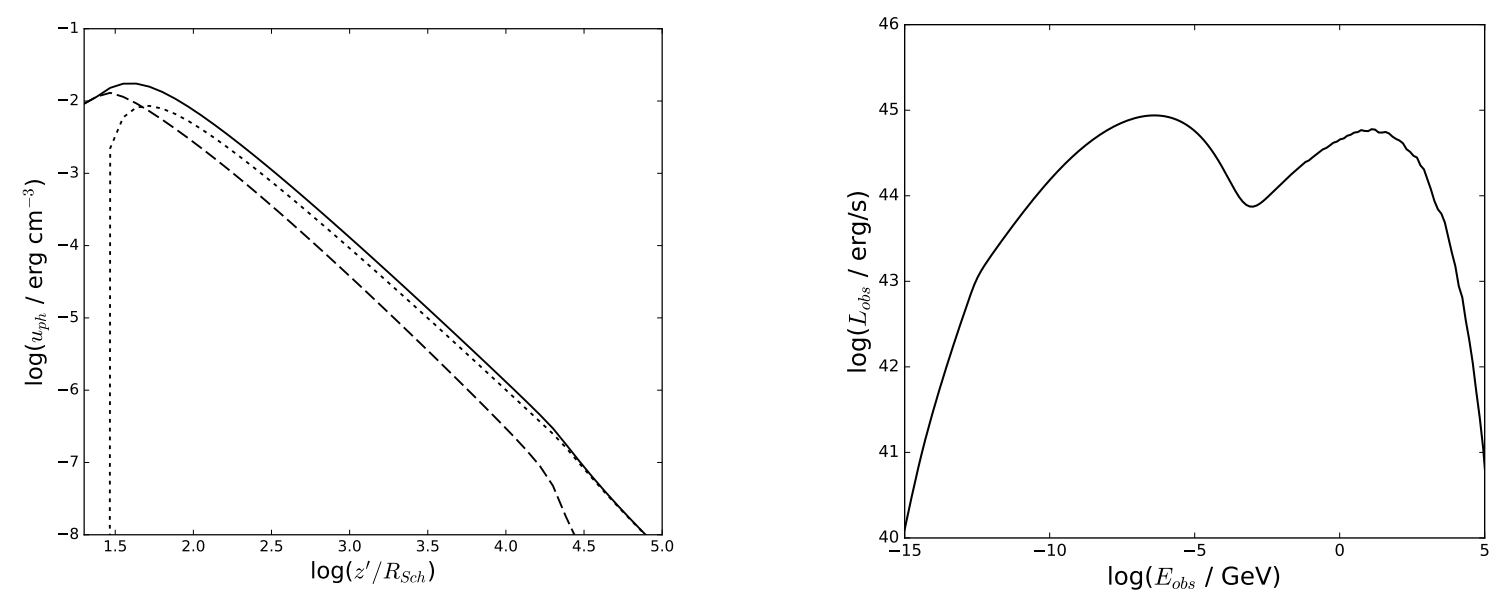

Figure 1: The energy density of synchrotron photons, produced in the extended jet, as a function of the distance from the jet base (on the left). The lines correspond to energy density of the backward photons (dotted line), the forward photons (dashed) and the total energy density of synchrotron photons (solid line). The spectral energy distribution (SED) from the extended jet (on the right). The parameters of the jet in both cases are $M_{B H}=10^{9} M_{\odot}, B_{0}=1 \mathrm{G}, \Gamma_{j}=5, L_{i n j}^{\prime}=10^{43} \mathrm{erg} / \mathrm{s}$, $\eta=3 \times 10^{-5}, z_{a c c}=100 R_{S c h}, \gamma_{\min }=1$.

$$
\begin{gathered}
n_{-}^{\prime}\left(\varepsilon^{\prime} ; t^{\prime}\right)=\frac{1}{c \varepsilon^{\prime}} \int_{z^{\prime}}^{z_{\max }^{\prime}} j^{\prime}\left(\varepsilon^{\prime}, \Omega ; z_{-}\right) \exp (-\tau) \Delta \Omega \mathrm{d} z_{-}, \\
n_{+}^{\prime}\left(\varepsilon^{\prime} ; t^{\prime}\right)=\frac{1}{c \varepsilon^{\prime}} \int_{z_{0}}^{z^{\prime}} j^{\prime}\left(\varepsilon^{\prime}, \Omega ; z_{+}\right) \exp (-\tau) \Delta \Omega \mathrm{d} z_{+},
\end{gathered}
$$

where $j\left(\varepsilon^{\prime}, \Omega ; z_{ \pm}\right)$is the synchrotron emissivity at the distance $z_{ \pm}$and $\tau=\tau_{S S A}$ is the optical depth for the self-synchrotron absorption (SSA) process. $\Delta \Omega$ is the solid angle of incoming radiation given by

$$
\Delta \Omega\left(z^{\prime}, z_{ \pm}\right)=\frac{2 \pi r h}{r^{2}}=2 \pi\left(1-\frac{\left|\left(z_{ \pm}-z^{\prime}\right)\right|}{r}\right),
$$

where $2 \pi r h$ is the area of the spherical layer and $r=\left[\left(z^{\prime}-z_{ \pm}\right)^{2}+R_{j}\left(z^{\prime}\right)^{2}\right]^{1 / 2}$ and $h=r-\left(z^{\prime}-z_{ \pm}\right)$.

The knowledge on the local density of photons and the differential spectrum of electrons, allow us to calculate the emissivities and absorption coefficients for the synchrotron, IC, synchrotron selfabsorption, and $\gamma-\gamma \rightarrow e^{ \pm}$pair creation processes in the plasma rest frame. Then, we obtain the observed luminosity of the jet by solving the equation of radiative transfer [12]. The example spectral energy distribution of a blazar jet is shown in Fig. 1.

\subsection{Cascades initiated by gamma-rays}

We use the model for radiation field produced in the stationary state of the large scale jet, obtained in previous section, to calculate the IC $\mathrm{e}^{ \pm}$pair cascade processes initiated by VHE $\gamma$-rays from the vicinity of the SMBH. We assume that the VHE $\gamma$-rays are injected at the jet base and pass through the extended jet. Such photons can be absorbed by the synchrotron photons whose density has been calculated with Eq. 2.2. As a result of the absorption, VHE $\gamma$-rays create electron-positron 


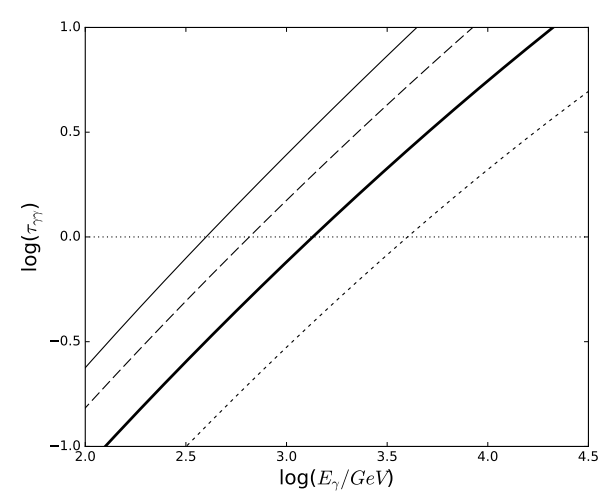

Figure 2: The optical depths for absorption of $\gamma$-rays in collisions with synchrotron photons produced in the extended, stationary jet. We show the optical depths for jets with different power injected into relativistic electrons (but the same other parameters) $L_{i n j}^{\prime}=3 \times 10^{42} \mathrm{erg} / \mathrm{s}$ (dotted line), $L_{\text {inj }}^{\prime}=10^{43} \mathrm{erg} / \mathrm{s}$ (thick solid), $L_{\text {inj }}^{\prime}=3 \times 10^{43} \mathrm{erg} / \mathrm{s}$ (dashed) and $L_{\text {inj }}^{\prime}=10^{44} \mathrm{erg} / \mathrm{s}$ (solid). The other parameters of the jet are listed in Fig. 1.

pairs. The $e^{ \pm}$pairs are cooled down on the synchrotron and the IC processes. It is possible that leptons emit a second generation of $\gamma$-rays. These $\gamma$-rays can be again absorbed or escape from the jet. For the sake of simplicity, we reduce the cascade to one dimension, i.e. the directions of the photons are close to the jet axis. Produced $e^{ \pm}$pairs are advected by the plasma inside jet. They are isotropized by the random component of the magnetic field.

The $\gamma$-ray injection rate, $Q_{H E}(\varepsilon, t)$, is described the distribution of VHE photons injected to the jet at the base at the time $t$. We consider three different forms for $Q_{H E}$ : (1) the injection of monoenergetic $\gamma$-rays; (2) the injection of $\gamma$-rays with the power law spectrum, and (3) injection of monoenergetic $\gamma$-rays in a broad interval time $(\delta t \sim 10 \mathrm{~min})$.

VHE $\gamma$-rays are absorbed in the synchrotron radiation of the jet. Since $\gamma$-rays propagate along the jet, the absorption occurs mainly when the HE photons interact with the forward photons $\left(n_{-}\right)$. In Fig. 2 we show the optical depths for $\gamma$-rays in the stationary jet. Photons with energy higher than $\geq 1 \mathrm{TeV}$ are absorbed efficiently. They initiate cascades inside the jet. The absorption threshold $\left(\tau_{\gamma \gamma}=1\right)$ is very sensitive on the jet power. For powerful jets, even the photons with energies above $300 \mathrm{GeV}$ are absorbed. $e^{ \pm}$pairs are created in the whole volume of the jet. We simulate the place of $e^{ \pm}$pair creation within the jet using the inverse transformation method. Similarly, the energies of $\mathrm{e}^{ \pm}$pairs are determined. It is assumed that the $e^{ \pm}$pairs are isotropized by the random component of the magnetic field (in the plasma rest frame). In order to describe IC process of pairs, we use the formula for the scattering of mono-directional photons on the isotropic distribution of relativistic electrons [13]. We accept that electrons lose energy continuously on the synchrotron and the IC processes in the Thomson regime. On the other hand, in the Klein-Nishina regime, the electrons lose energy in discrete amounts producing $\gamma$-rays with specific energies.

\section{Expected cascade spectra}

We examine the injection of VHE $\gamma$-rays into the volume of the stationary jet as described in Section 2.1. We apply the typical parameters of the stationary jet as mentioned above, i.e. the mass 

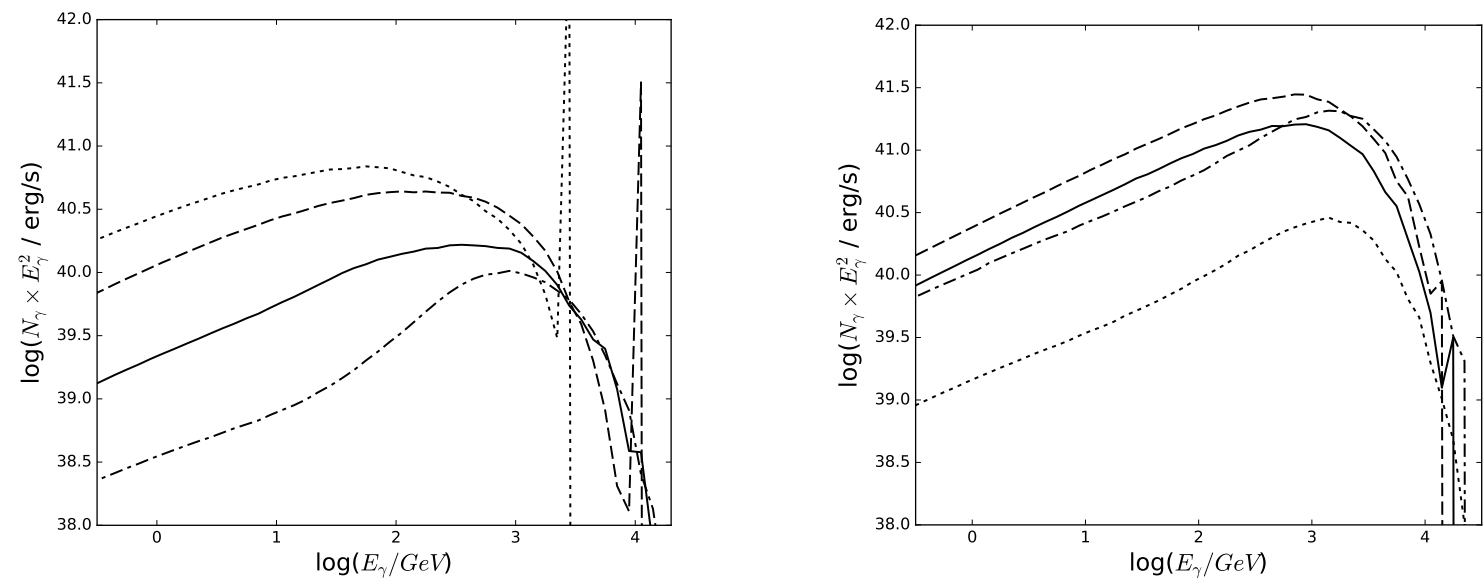

Figure 3: $\gamma$-ray spectra observed from the IC $e^{ \pm}$cascades for different initial photons distributions. The cascades are initiated by monoenergetic $\gamma$-rays with energy $3 \mathrm{TeV}$ (dotted line), $10 \mathrm{TeV}$ (dashed), $30 \mathrm{TeV}$ (solid) and $100 \mathrm{TeV}$ (dot-dashed), on the left. The secondary $\gamma$-ray spectra are shown for the primary $\gamma$-rays with the power low distribution for spectral index $s=1.0$ and $s=1.5$ (on the right). The parameters of the jet are listed in Fig. 1.

of the black hole $M_{B H}=10^{9} M_{\odot}$, the magnetic field at the base of the jet $B_{0}=1 \mathrm{G}$, the bulk Lorentz factor of the jet $\Gamma_{j}=5$, the total power injected in relativistic electrons into the jet $L_{i n j}^{\prime}=10^{43} \mathrm{erg} / \mathrm{s}$, the acceleration coefficient $\eta=3 \times 10^{-5}$, the place of the acceleration starting at $z_{a c c}=100 R_{\text {Sch }}$ and the minimum Lorentz factor of injected electrons $\gamma_{\min }=1$.

Firstly, we investigate the case of the monoenergetic beam of $\gamma$-rays passing the synchrotron photon field of the jet. In Fig. 3 (on the left), we show the spectra of $\gamma$-rays after propagation through the jet radiation field. The monoenergetic $\gamma$-rays in the energy range from $3 \mathrm{TeV}$ up to 100 $\mathrm{TeV}$ are considered. The power in injected $\gamma$-rays in every case is $L_{H E}=10^{43} \mathrm{erg} / \mathrm{s}$ (in observer rest frame). The $\gamma$-ray spectra, produced in the cascade, depend strongly on the energy of the primary photons. The cut-offs in these spectra are shifted to the higher energies for the more energetic primary $\gamma$-rays. The power emitted in the cascade $\gamma$-rays are higher for the primary $\gamma$-rays with smaller energies. This is due to the synchrotron cooling of $e^{ \pm}$pairs. For the primary $\gamma$-rays with low energies, 3 and $10 \mathrm{TeV}$, the secondary spectra extend through the relatively narrow energy range. This effect is caused by the non-absorbed initial $\gamma$-rays.

In Fig. 3, we show spectra for the power law distribution of primary photons with the spectral indices 1.5 and 1.0 and two maximum energies of the cut-offs in those spectra at $30 \mathrm{TeV}$ and 300 $\mathrm{TeV}$. The cut-offs in the secondary $\gamma$-ray spectra are located at similar energies. Similarly to the cases with the injection of the monoenergetic primary $\gamma$-rays, the power emitted in secondary $\gamma$ ray spectra is smaller for larger maximum energies of primary $\gamma$-rays. It should be noted, that the obtained spectral shapes are independent on the spectral index.

For the jets pointed toward the observer $\left(\theta_{o b s} \sim 0^{\circ}\right)$, the expected time duration of a flare due to the development of the IC $e^{ \pm}$cascades is similar to the injection time. We show the light curve for radiation produced in the IC $e^{ \pm}$cascade in Fig. 4 (on the left). The injection time of the VHE $\gamma$-rays is $t_{i n j}=600 s$. The spectrum of photons is monoenergetic with the characteristic energy of $E_{\gamma}=30 \mathrm{TeV}$. The rising stage, when the new photons are continuously created in the cascades, is 

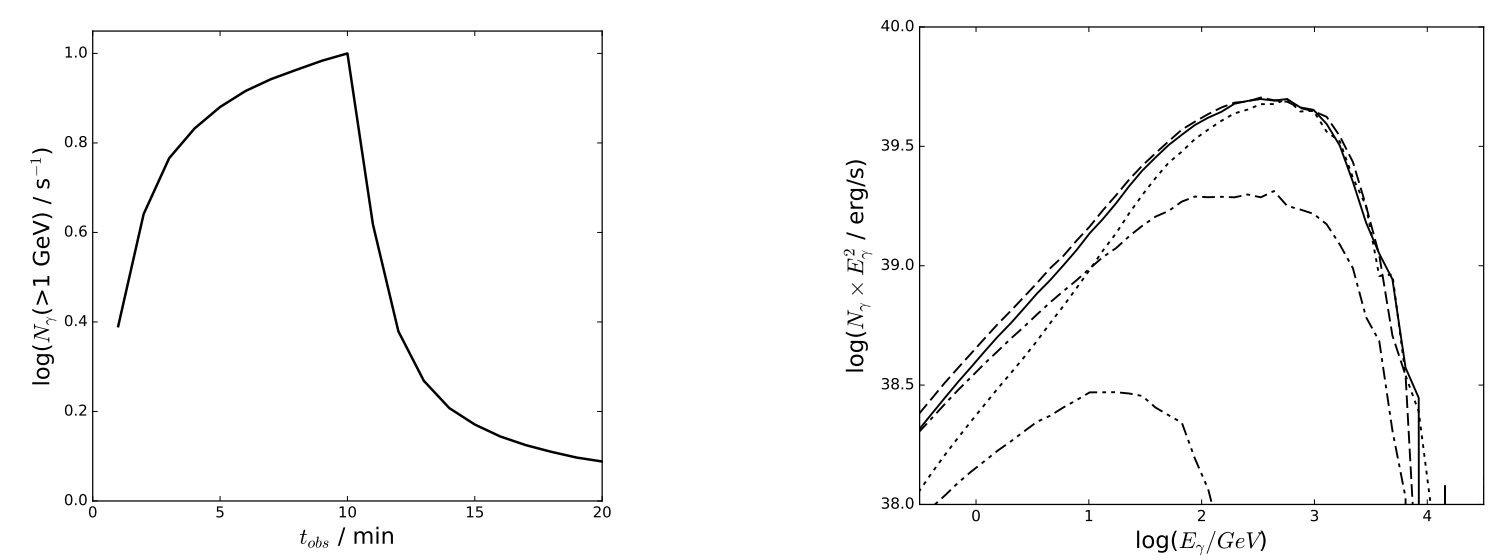

Figure 4: The normalized number of photons, with energy higher than $1 \mathrm{GeV}$, produced in the IC $e^{ \pm}$cascades initiated by the monoenergetic beam of primary $\gamma$-rays with energy $E_{\gamma}=30 \mathrm{TeV}$ (on the left). The evolution of SED produced in the IC $e^{ \pm}$cascades propagated in the extended jet (on the right). The lines correspond to different observation time intervals: 0-3 min (dotted line), 3-6 $\min$ (solid), 6-9 $\mathrm{min}$ (dashed), 9-12 $\mathrm{min}$ (dot-dashed) and 12-15 $\mathrm{min}$ (dot-dot-dashed). The energy of primary $\gamma$-rays are equal to $E_{\gamma}=30 \mathrm{TeV}$. The parameters of the jet are listed in Fig. 1.

determined by the injection of primary $\gamma$-rays. The decaying phase of the flare is determined by the cooling processes of leptons. In this same figure (on the right), we present the evolution of the SED during and after the injection of primary $\gamma$-rays. During the injection the spectra are similar. Their cutoffs are located approximately at the absorption threshold. Just after the injection, the spectra decrease quickly with time. The peaks in the spectra shifts to lower energies.

\section{Conclusion}

We have developed the model of the parsec-scale jet in a low emission state. It is assumed that the radiation in such a state is produced in an extended part of the jet. We show that this model predicts reasonable fits to the spectra of blazars with the typical parameters describing the jet. Using the knowledge about the radiation field inside the jet, we conclude that the considered jet is opaque for $\gamma$-rays with energies larger than a few TeV. In the case of the most powerful jets, the jet becomes opaque fro $\gamma$-rays already at $\mathrm{GeV}$ energies. The optical depths through the jet increase with energy of $\gamma$-rays. It depends strongly on the jet power.

If the jet is non-transparent for $\mathrm{TeV}$ photons produced in the vicinity of the $\mathrm{SMBH}$, they should be absorbed on the synchrotron radiation of the extended jet. This is especially important for blazars, in which the observation angle is very small. Then, $\gamma$-rays must first pass through the whole volume of the jet. We have investigated the opacity for $\gamma$-rays with the monoenergetic and very hard power law spectra in the extended jet. The absorbed $\gamma$-rays create a high energy secondary $e^{ \pm}$pairs which develop the IC $\mathrm{e}^{ \pm}$pair cascades in the volume of the jet. We have shown the secondary $\gamma$-ray spectra from such cascades initiated by the monoenergetic $\gamma$-rays and also $\gamma$ rays with the power law distribution. The place of the cut-off in the secondary spectra is determined by their absorption threshold in the jet radiation. We have shown that for very small observation 
angles $\left(\theta \sim 0^{\circ}\right)$, the time duration of the emission from the IC $\mathrm{e}^{ \pm}$pair cascades is similar to the injection time of the primary $\gamma$-rays although the cascades develop through the large part of the jet.

\section{Acknowledgments}

The research has been supported by the Polish NCN grant 2015/19/N/ST9/01727

\section{References}

[1] F. Aharonian, et al., An Exceptional Very High Energy Gamma-Ray Flare of PKS 2155-304, ApJ 664 (2007) L71

[2] J. Albert, et al., Variable Very High Energy Îş-Ray Emission from Markarian 501, ApJ 669 (2007) 862

[3] J. Aleksić, et al., Black hole lightning due to particle acceleration at subhorizon scales, Science 346 (2014) 1080

[4] A. Donea, R. Protheroe, Radiation fields of disk, BLR and torus in quasars and blazars: implications for $\gamma$-ray absorption, APh 18 (2003) 377

[5] A. Neronov, F. Aharonian, Production of TeV Gamma Radiation in the Vicinity of the Supermassive Black Hole in the Giant Radio Galaxy M87, ApJ 671 (2007) 85

[6] K. Hirotani, et al.,Lepton Acceleration in the Vicinity of the Event Horizon: High-energy and Very-high-energy Emissions from Rotating Black Holes with Various Masses, ApJ 883 (2016) 142

[7] W. Bednarek,Gamma-rays from synchrotron pair cascades in blazars?, MNRAS 285 (1997) 69

[8] D. Giannios, et al.,Fast TeV variability in blazars: jets in a jet, MNRAS 395 (2009) L29

[9] A. Pushkarev, et al., Apparent parsec-scale jet opening angles and gamma-ray brightness of active galactic nuclei, (arXiv:1205:0659)

[10] E. Clausen-Brown, et al.,Causal connection in parsec-scale relativistic jets: results from the MOJAVE VLBI survey, A\&A $\mathbf{5 5 8}$ (2013) A114

[11] M. Chiaberge, G. Ghisellini,Rapid variability in the synchrotron self-Compton model for blazars, MNRAS 306 (1999) 551

[12] K. Lind, R. Blandford,Semidynamical models of radio jets - Relativistic beaming and source counts, ApJ 295 (1985) 358

[13] F. Aharonian, A. Atoyan,Compton scattering of relativistic electrons in compact X-ray sources, Ap\&SS 79 (1981) 321 